Abstract PS4:72 Table 1

\begin{tabular}{|l|c|c|r|}
\hline & $\begin{array}{c}\text { AlHA (\%) } \\
\mathbf{n}=16\end{array}$ & $\begin{array}{c}\text { CONTROLS (\%) } \\
\mathbf{n = 3 2}\end{array}$ & p value \\
\hline aPL positivity & $13(81)$ & $14(44)$ & $\mathbf{0 , 0 1 6 4}$ \\
\hline Single aPL positivity & $2(13)^{*}$ & $4(13)$ & 1,0000 \\
\hline Double aPL positivity & $8(53)^{*}$ & $7(22)$ & $\mathbf{0}, 0459$ \\
\hline Triple aPL positivity & $1(7)^{*}$ & $3(9)$ & 1,0000 \\
\hline aCL & $6(39)$ & $8(25)$ & 0,5026 \\
\hline aCL IgG & $4(25)$ & $8(25)$ & 1,0000 \\
\hline low-titer & $2(13)$ & $2(6)$ & 0,5921 \\
\hline medium-titer & $2(13)$ & $5(16)$ & 1,0000 \\
\hline high-titer & $0(0)$ & $1(3)$ & 1,0000 \\
\hline aCL IgM & $4(25)$ & $4(13)$ & 0,4130 \\
\hline low-titer & $3(19)$ & $3(9)$ & 0,3858 \\
\hline medium-titer & $1(6)$ & $1(3)$ & 1,0000 \\
\hline high-titer & $0(0)$ & $0(0)$ & 1,0000 \\
\hline $\boldsymbol{\beta}_{2}$ GPI & $10(63)$ & $13(41)$ & 0,2221 \\
\hline $\boldsymbol{\beta}_{2}$ GPI IgG & $8(50)$ & $10(31)$ & 0,2258 \\
\hline low-titer & $6(39)$ & $6(19)$ & 0,1776 \\
\hline medium-titer & $2(13)$ & $1(3)$ & 0,2544 \\
\hline high-titer & $0(0)$ & $3(9)$ & 0,5412 \\
\hline $\boldsymbol{\beta}_{2}$ GPI IgM & $6(39)$ & $6(19)$ & 0,1776 \\
\hline low-titer & $4(25)$ & $5(16)$ & 0,4566 \\
\hline medium-titer & $2(13)$ & $0(0)$ & 0,1064 \\
\hline high-titer & $0(0)$ & $1(3)$ & 1,0000 \\
\hline IgG (aCL+ $\boldsymbol{\beta}_{2}$ GPI) & $12(75)$ & $18(56)$ & 0,3431 \\
\hline IgM (aCL+ $\boldsymbol{\beta}_{2}$ GPI) & $10(63)$ & $10(31)$ & 0,5908 \\
\hline LAC & $8(50)^{*}$ & $5(16)$ & 0,0131 \\
\hline & & &
\end{tabular}

${ }^{*} \mathrm{n}=15$

anticoagulant (LAC) as other highlighted the association with a positive anti-B2glycoprotein I (B2GPI) IgG or anti-cardiolipin (aCL) IgM. The purpose of our preliminary study is to analyse and compare aPL positivity in two groups of SLE patients, one with a history of AIHA and one without it.

Methods The retrospective analysis of our cohort of 514 SLE patients showed that 16 subjects fulfil a completely documented diagnosis of AIHA at some stage of their disease. These subjects and 32 consecutive SLE patients without any haematological manifestations were included in this study. Clinical and serological data were obtained from medical records of our clinic. We compared the prevalence of aPL, their type (aCL IgG and IgM, B2GPI IgG and IgM, LAC) and titre using Fisher exact test in the two groups of patients.

Results Lupus patients with AIHA had a significantly higher frequency of aPL positivity compared with SLE patients without AIHA (81\% vs 44\%, p value=0,0164). Furthermore, double aPL positivity was detected in $53 \%$ of patients with AIHA as in the control group was $22 \%$ ( $p$ value $=0,0459$ ). The positivity of aCL and B2GPI was higher in the group of patients with history of AIHA than in the control group (39\% vs $25 \%$ and $63 \%$ vs $41 \%$, respectively) but the difference was not statistically significant; table1 details the results of each antibody isotype and titres. The presence of LAC was significantly associated with history of AIHA ( $p$ value $=0,0131$ ).
Conclusions In this control-case study the frequency of aPL was higher in SLE patients with a history of AIHA. In particular, in this group we found a higher frequency of double aPL positivity. Between aPL, LAC had the strongest association with AIHA. The absence of statistical difference considering aCL and B2GPI positivity and titres could be due to the low sample size.

\section{PS4:73 EFFECT OF HYDROXYCHLOROQUINE AND PREDNISONE TREATMENT ON ANTIPHOSPHOLIPID TITERS IN SLE}

${ }^{1} \mathrm{M}$ Avci, ${ }^{2} \mathrm{~L}$ Magder, ${ }^{3} \mathrm{M}$ Petri. ${ }^{1}$ Istanbul Faculty of medicine, Istanbul, Turkey; ${ }^{2}$ University of Maryland School of Medicine, Baltimore, MD, USA; ${ }^{3}$ Johns Hopkins University School of Medicine, Baltimore, MD, USA

\subsection{6/lupus-2018-abstract.119}

Background Antiphospholipid antibodies in SLE may be affected by disease activity and treatment, but past studies have been conflicting. We investigated the association of titers of antiphospholipid antibodies with disease activity and therapy.

Methods 943 SLE patients, who had at least 10 quarterly visits for testing for each anticardiolipin isotype (IgG, $\operatorname{IgM}$ and IgA) and dRVVT, were included in the study. Disease activity 
was measured with both the Systemic Lupus Erythematosus Disease Activity Index (SLEDAI) and Physician Global Assessment (PGA). Treatment was recorded at every visit.

Results aCL IgG $>40$ and aCL IgA were not associated with disease activity. Hydroxychloroquine reduced the levels of all antibodies, except for IgA aCL. Prednisone reduced aCL IgG but not aCL IgM, aCL IgA $>40$ or dRVVT (seconds prolongation).

Conclusion High titer aCL were not affected by disease activity. Thus these patients are more like 'primary' antiphospholipid patients. Hydroxychloroquine use was associated with reduced lupus anticoagulant (by seconds of prolongation) and reduced titers for most of the isotypes of anticardiolipin. Prednisone did not reduce the seconds of dRVVT prolongation. Anticardiolipin IgA seemed the most resistant to therapy.

\section{PS4:74 PRIMARY ANTIPHOSPHOLIPID SYNDROME PRESENTING WITH PURPURA FULMINANS - THERAPEUTIC RESPONSE TO GLUCOCORTICOIDS, ANTICOAGULATION AND PLASMA EXCHANGE}

M Plüß, M Zeisberg, GA Müller, P Korsten. University Medical Centre Göttingen, Department of Nephrology and Rheumatology, Göttingen, Germany

\subsection{6/lupus-2018-abstract.120}

Purpose Antiphospholipid syndrome (APS) is a severe and potentially life-threatening condition. Most commonly, it presents with arterial or venous thrombotic complications. Unusual presentations might lead to a delay in diagnostic and therapeutic measures. Purpura fulminans (PF) is a rarely reported manifestation.

Methods Case report at a single centre.

Results We present the case of a 25-year-old female who developed purpura fulminans after evacuation of a perimandibular abscess. The patient was initially admitted to an external maxillofacial surgery unit for incision and drainage of a perimandibular abscess after multiple episodes of tonsillitis. Her past medical history includes a significant spontaneous deep venous thrombosis 8 months prior to admission. APS had been suspected in view of one pathological lupus anticoagulant result, but a diagnosis had not been made at the time. The patient was anticoagulated with Rivaroxaban and had experienced no further events. Lupus serology remained negative. Immediately after maxillofacial surgery, the patient developed lightning bolt-shaped hematomas with peripheral blisters and central necrosis on both arms, her right thigh, the back of her neck and torso, as well as both earlobes.

She was diagnosed with PF and transferred to our rheumatology unit, where her medical management was adjusted with a switch from oral anticoagulation to low-molecular weight heparin and initiation of oral corticosteroid therapy. Further testing confirmed the working diagnosis of APS: a pathological lupus anticoagulant, raised anti-cardiolipin $\operatorname{IgM}$ and $\mathrm{IgG}$, positive beta- 2 glycoprotein, high aPTT, and low complement C3. A skin biopsy demonstrated multiple thrombi in blood vessels of the dermis. In light of this result and further clinical deterioration of the PF even under oral steroid therapy, treatment was escalated to five sessions of plasma exchange. With this treatment, the patient's condition improved drastically. There were no further purpura or blisters and the existing skin lesions lightened and grew smaller. Anticoagulation was switched from heparin to Warfarin and the patient was discharged on a tapering dose of oral Prednisolone.

Conclusion PF is a rare presentation of APS. This case demonstrates the value of early and resolute escalation of treatment. Plasma exchange is a potentially life-saving treatment modality in this setting.

\section{PS4:76 THE CHALLENGE OF CENTRAL NERVOUS SYSTEM SMALL VESSEL DISEASE IN AUTOIMMUNITY: A CASE REPORT}

${ }^{1} \mathrm{~L}$ Duarte, ${ }^{1} \mathrm{MP}$ Anselmo, ${ }^{1} \mathrm{C}$ Mendes, ${ }^{1} \mathrm{C}$ Mota, ${ }^{1} \mathrm{JM}$ Lopes, ${ }^{1} \mathrm{~J}$ Meneses, ${ }^{2} \mathrm{R}$ Victorino. ${ }^{1} \mathrm{CHLN}$ - Hospital Santa Maria - Department of Internal Medicine II - A. Lisboa, Portugal; ${ }^{2}$ CHLN - Hospital Santa Maria - Department of Internal Medicine II, Lisboa, Portugal

10.1136/lupus-2018-abstract.121

Among neurological involvement of autoimmune diseases, microvasculopathy and small vessel disease remains quite challenging, with an unclear aetiology and heterogeneous clinical manifestations. A 54-year-old male was admitted at the Emergency Department complaining of motor deficit of right hand since the day before. Neurological examination revealed clumsy hand with difficulty in writing, with ataxia and dysdiadochokinesia of the right arm. Magnetic resonance imaging revealed probable central nervous system (CNS) vasculitis, with multiple acute and chronic micro ischaemic lesions without respecting a single arterial territory, in both cerebral and cerebellar hemispheres. Delving into his personal medical records reference should be made to the fact that he has already had thrombotic events in the previous six years, twice deep vein thrombosis and myocardial infarction, recurrent oral ulcers and no suggestion of kidney disease. Laboratory results showed pancytopenia, creatinine: $2,7 \mathrm{mg} / \mathrm{dL}$; BUN: $102 \mathrm{mg} / \mathrm{dL}$; ESR: $77 \mathrm{~mm} / \mathrm{h}, \mathrm{CRP}$ : 2,3 mg/dL, LDL cholesterol: $120 \mathrm{mg} / \mathrm{dL}$, HbA1c: 5,4\%, homocysteine: $28,5 \mu \mathrm{mol} / \mathrm{L}$, haptoglobin: $161 \mathrm{mg} / \mathrm{dL}$. A broader laboratory study was made, including measure of anticardiolipin antibodies - IgG (6842,6 UQ) and IgM (20-30 UQ), antibeta2-glycoprotein-I antibodies - IgG (22298,7 UQ) and IgM (20-30 UQ) positive lupus anticoagulant test, Anti-DS-DNA antibodies: 55,2 UI/mL, low $\mathrm{C} 3$ and direct Coombs test: IgG positive, C3d negative. 24 hour urine sample showed proteinuria of $1859,7 \mathrm{mg} / 24$ hour and an Albumin-Creatinine ratio of $1019,7 \mathrm{mg} / \mathrm{g}$. Transthoracic echocardiogram, Holter monitoring, and echo-Döppler ultrasound of neck vessels did not highlight any abnormality. Kidney biopsy demonstrated mesangial proliferative glomerulonephritis and didn't show the typical 'full house' immunofluorescence of systemic lupus erythematosus (SLE), rather glomerular mesangial widening and hypercellularity and focal glomerular capillary wall thickening with double contours, intraluminal thrombi and endothelial cell swelling. At the moment, the patient presents symptoms that fulfil the current diagnostic criteria for secondary antiphospholipid syndrome (APS) to SLE, due to 9 of SLICC criteria presented. Even so, the difficulty in defining the aetiology of the involvement of the CNS persists, considering the possibility of neurolupus or CNS vasculitis associated with APS. This case illustrates the clinical challenge of distinguishing CNS small vessel disease in the context of autoimmunity. 